\title{
How we use Facebook to achieve our goals: a priming study regarding emotion regulation, social comparison orientation, and unaccomplished goals
}

\author{
Phillip Ozimek ${ }^{1}$ (D) Hans-Werner Bierhoff ${ }^{2} \cdot$ Katharina M. Hamm $^{2}$ \\ Published online: 25 June 2020 \\ (C) The Author(s) 2020
}

\begin{abstract}
On the basis of recent findings in the context of the Social Online Self-Regulation Theory it is assumed that intensity of social Facebook use (i.e., a higher intensity and more time spend on Facebook focusing on social activities) serves the purpose of selfregulation. A priming of unfulfilled goals was used to increase participants' intensity of social Facebook use in order to regulate the negative emotions and the self-discrepancy which should be caused by the priming. High social comparison orientation as well as difficulties in emotion regulation were assumed to moderate that effect. 355 people took part in an online survey. As expected, difficulties in emotion regulation as well as social comparison orientation were positively correlated with intensity of social Facebook use. However, the priming effect was moderated by difficulties in emotion regulation. Our research follows propositions derived from self-regulation theory and is, therefore, integrated into a broader theoretical approach focusing on the goal-setting of the self. Finally, possible applications of this research on the explanation of dysfunctional social Facebook use are outlined.
\end{abstract}

Keywords Facebook $\cdot$ Self-regulation $\cdot$ Social comparison theory $\cdot$ Emotion regulation $\cdot$ Goal striving

\section{Introduction}

I begin with the egoistic premise that one's highest moral purpose is the achievement of one's own happiness (Locke, 2001).

The social network Facebook has been known worldwide for several years now, influencing the lives of more than two billion users (Roth, 2018). Founded in 2004, it was originally designed for Harvard students only, before it expanded and became increasingly popular around the world. In 2017, Marc

Phillip Ozimek

phillip.ozimek@ fh-muenster.de

Hans-Werner Bierhoff

hans.bierhoff@rub.de

Katharina M. Hamm

katharina.hamm@rub.de

University of Applied Sciences Münster, Münster, Germany

2 Ruhr-University Bochum, Bochum, Germany
Zuckerberg announced Facebook's new mission to “give people the power to build community and bring the world closer together" (Zuckerberg, 2017). People are assumed to use Facebook in order to build network, stay informed, and, at the same time, inform others about what is important to them. To put it simply, Facebook is supposed to make people happy.

Social scientists around the world address the phenomenon of Facebook (see Wilson, Gosling, \& Graham, 2012 for a review) and its effects on users. Facebook serves goals which are positively related to users' health. Specifically, empirical studies indicate positive effects on health, self-esteem, and other areas of life (e.g., Kim \& Lee, 2011; Toma, 2013; Nabi, Prestin, \& So, 2013). In contrast, other empirical findings question the idea of Facebook making people happy. Indeed, numerous studies found negative consequences of Facebook use focusing on social activities, such as lower self-esteem, less life satisfaction, and more depressive symptoms (e.g., Kross et al., 2013; Steers, Wickham, \& Acitelli, 2014). So why do people keep using Facebook?

Research revealed various motives and reasons for the usage and popularity of the social network site (SNS) Facebook, e.g., the need to belong and the need for self-presentation (Nadkarni \& Hofmann, 2012). These needs, which are independent from each other, are influenced by a variety of determinants including culture, personality, and sociodemographic 
variables. Two competing hypothesis emphasize either enhancement of success or compensation of failure as primary factors which motivate use of Facebook and other SNSs as well: According to the Social Enhancement Hypothesis ("rich get richer"), people who are very popular offline use Facebook to become even more popular online, while the Social Compensation Hypothesis ("poor get richer") implies that offline unpopularity is being compensated online (cf., Valkenburg, Schouten, \& Peter, 2005). Although Facebook use may satisfy a broad variety of needs, we focus on social Facebook use which refers to the intensity and amount of Facebook use with respect to "social Facebook features", such as commenting on or looking at others' profiles, sending private messages, communicating in Facebook groups and so on. The need to belong, the need to self-presentation, and the need for social comparison are each related to social Facebook use. Of course, Facebook serves a manifold of goals beyond social activities such as gaming or advertising. But our proposition is that the main motive for using Facebook with respect to social activities as means for emotion regulation as well as for general self-regulation is based on a social perspective.

Furthermore, personality variables might influence intensity of social Facebook use. Previous studies have shown that social Facebook use is, among other factors, associated with dispositional interest in social comparisons (Appel, Gerlach, \& Crusius, 2016; Ozimek \& Bierhoff, 2016; Verduyn et al., 2017). Personality traits including materialism, extraversion, narcissism, conscientiousness, neuroticism, loneliness, and shyness were also linked with individual differences in Facebook use (Brailovskaia \& Bierhoff, 2016; Ryan \& Xenos, 2011; Ozimek, Baer, \& Förster 2017; Ozimek, Bierhoff, \& Hanke, 2018).

The Social Online Self-Regulation Theory (SOS-T; Ozimek, et al., 2017) unifies previous findings with respect to reasons for Facebook usage by uncovering fundamental motives behind it. The study at hand was planned as a test of the basic assumptions of the SOS-T, regarding emotion regulation and social comparison. Besides correlational data, experimental data were obtained which were based on a priming procedure to answer the question which effect the activation of unaccomplished goals has on the readiness to use Facebook. Examination of these data is likely to provide insight into the determinants of user behaviour on Facebook and its repercussions on the user as an individual.

\section{Theoretical Background}

\section{Facebook and Self-Regulation: The Social Online Self- Regulation Theory}

The SOS-T states that social networking sites, such as Facebook, are unconsciously used for self-regulation in order to reach certain goals and eventually hedonistic positive end states, i.e., to be happy. The theory assumes that Facebook offers high multifinality (see also Förster, Liberman, \& Friedman, 2007; Kruglanski et al., 2002), i.e., various goals can be reached by using it, and that, furthermore, the high popularity of Facebook and its easy accessibility make Facebook an attractive means to achieve a variety of goals and, ultimately, happiness. However, the theory does not imply functionality. People can unconsciously assume that social Facebook use facilitates a positive emotional outcome while in reality this consequence does not occur. The question whether social Facebook use really creates happiness or satisfaction is not in the focus of our study. Instead, the focus is on investigating how the unconscious conviction that Facebook contributes to the well-being of the users influences their online behaviour in general and their self-regulation of social Facebook use in particular. Self-regulation characterizes a process of controlling and planning one's own behaviour in order to reach desirable thoughts, positive affect, and personally relevant goals (Carver \& Scheier, 1982). The SOS-T describes a feedback loop in which the current situation is continuously compared to a norm or reference point of the desirable condition. In case of the detection of deviations from the reference point, an action is initiated which serves to restore the desirable condition. On the highest level, the ideal self which is defined as own or someone else's "hopes, goals, or wishes for the person" (Higgins, Strauman, \& Klein, 1986, p. 6) serves as reference point. SOS-T is closely related to the Self Discrepancy Theory (Higgins, Klein, \& Strauman, 1985) which states that a perceived deviation of the actual self from this ideal self can potentially elicit "dejection-related emotions and symptoms" such as disappointment and dissatisfaction (p. $1)$.

Such negative emotions are likely to occur when personal goals are not achieved (Kruglanski et al., 2002). Goals can be defined as ,future-oriented representations of what individuals are striving for in their current life situations and what they try to attain or avoid in various life domains" (Brunstein, Dangelmayer, \& Schultheiss, 1996; Brunstein \& Maier, 1996; cited by Brunstein, Schultheiss, \& Maier, 1999, p. 171). Goal systems, which are hierarchical organized, are crucial for the understanding of the process of self-regulation: "For example, shopping can be a means to the end of gaining status and further gaining status may be a means to the end of becoming happy" (Ozimek \& Förster, 2017, p. 419).

A frequently used method of goal activation in the context of self-regulation is the pre-activation of goals by priming procedures (cf., Förster \& Jostmann, 2015; Förster \& Liberman, 2007). Because self-regulation tends to occur unconsciously (Förster \& Jostmann, 2015; see also Kruglanski et al., 2002; Gross, 1998), corresponding priming techniques are promising as a means of activating it. Important rules to assess robust and "clean" priming effects are that participants do not see a connection between priming and dependent 
variables and that the dependent variables are assessed immediately after the priming task because priming effects tend to rapidly vanish (for a more comprehensive review see Kruglanski et al., 2002).

Interestingly, recent priming studies with respect to selfregulation on Facebook have shown that the priming of specific goals and needs (i.e., high materialistic concerns and a high social comparison orientation; cf. Ozimek \& Förster, 2017) leads to a higher need for social Facebook use. Consideration of emotion regulation might provide a significant step toward understanding the mechanisms which activate an interest in social Facebook use. Former studies with respect to Facebook and self-regulation did not take into account that besides personality variables (e.g., high materialistic concerns or a high social comparison orientation) emotion regulation represents a crucial part of self-regulation processes. Therefore, emotion regulation was included in the analysis.

\section{Facebook and Emotion Regulation}

Emotion regulation refers to the processes by which individuals influence which emotions they have, when they have them, and how they experience and express these emotions (Gross, 1998, p. 275).

According to Gross, such processes either run automatically or controlled, i.e., unconsciously or consciously. It can be done by increasing or decreasing emotions and both positive and negative emotions can be regulated (Gross \& Thompson, 2007), although negative ones might be regulated more often (Gross, Richards, \& John, 2006). Some researchers distinguish between conscious and unconscious forms of emotion regulation (e.g., Masters, 1991). Gross (1998) distinguishes five forms of emotion regulation, i.e., cognitive change, situation modification, response modulation, situation selection, and attentional deployment. He states that emotion regulation can be antecedent-focused, which means it sets in before the emotional response, or response-focused, which happens after the emotional response. For example, attentional deployment can happen in the form of withdrawing the attention physically (e.g., by covering one's ears or eyes), distracting or concentrating, or it can be the response to other people redirecting a person's attention (Gross et al., 2007). These forms of emotion regulation are already exhibited by small children (Mischel \& Ayduk, 2004). Perhaps Facebook use in itself could be a kind of emotion regulation. This assumption is supported by Ryan, Chester, Reece, and Xenos (2014) who stated that people can use Facebook for altering their mood, which could be a sign for Facebook addiction as well. Ozimek et al. (2017) found that Facebook is an attractive, easy, and fast medium for achieving positive affect because it offers various possibilities of self-presentation, such as status updates or photos, while at the same time offering direct positive feedback in the form of likes or comments (see also Gonzales \& Hancock, 2011).
Thus, not only a distinct form of emotion regulation, but also emotion regulation in general can be realized by using Facebook. As Facebook enables everyone to present themselves, it will nearly inevitably cause people to compare themselves and their own self-presentation to those of other users: the process of social comparison.

\section{Social Comparison and Facebook}

Social Comparison Theory (Festinger, 1954) assumes the existence of a fundamental tendency of individuals to compare their own point of view and achievements in terms of progress in different aspects of life to the results of others in order to be able to classify themselves more precisely and meaningfully. It is suspected that social comparisons fulfil basic human motives such as maintaining and enhancing self-esteem (Mehdizadeh, 2010; Steinfield, Ellison, \& Lampe, 2008). In addition, a personality trait is connected to social comparisons which is called social comparison orientation varying between different individuals as well as between genders and cultures (Gibbons \& Buunk, 1999; Guimond et al., 2007). It is possible to compare oneself with a higher or lower standard, depending on which motive stands behind the comparison. An upward directed comparison serves as an incentive which elicits a focus on how to improve performance (Bandura, 1986). This function of upward comparisons is only realized when it is realistically possible for the person to deliver an improved performance (Lockwood \& Kunda, 1997). On the other hand, Wills' (1981) Theory of Downward Comparison Principles states that self-esteem might also be enhanced successfully by downward directed comparisons. Gibbons and Gerrard (1989) supported that when presented information about a person who is coping with a situation worse than themselves participants with low self-esteem experienced improved mood states.

With Facebook as the nearly ideal platform for performing social comparisons, it seems plausible that some people access the social online network in order to improve their mood state via social comparison. In correspondence with such an assertion many researchers (e.g., Appel et al., 2016; Lee, 2014; Ozimek \& Bierhoff, 2016; Ozimek et al., 2017, 2018; Vogel, Rose, Okdie, Eckles, \& Franz, 2015; Verduyn et al., 2017), found a positive correlation between social comparison orientation and intensity of Facebook usage. However, there are also findings indicating associations between social comparison and negative emotions (e.g., Chou \& Edge, 2012; Vogel et al., 2015), including low self-esteem (Mehdizadeh, 2010; Steinfield, Ellison, \& Lampe, 2008; Verduyn et al., 2017) and feelings of loneliness (Ryan \& Xenos, 2011). Therefore, it is unclear whether goals such as increasing self-esteem are indeed achieved by Facebook users, or if the need for enhancing self-esteem is merely a motivation for accessing Facebook which is not satisfied in reality (Ellison, Steinfield, \& 
Lampe, 2007; Valkenburg et al., 2006). In any case, the need for social comparison is seen as the third motive for using Facebook, next to the need to belong and the need for selfpresentation (cf. Nadkarni \& Hofmann, 2012; Ozimek \& Bierhoff, 2016).

The $S O S-T$ states that social comparison orientation as a motive activates certain goals and, depending on these goals, people may use social media as a means to their personal ends (see Ozimek et al., 2017). A "high social comparer" could use Facebook to satisfy her need to compare herself to important others (cf. Lee, 2012; Ozimek \& Bierhoff, 2016; Ozimek \& Förster, 2017). In addition, personality traits like narcissism, materialism, and self-esteem activate specific goals. These activated goals can be manifold and diverse. E.g., a narcissist may use Facebook to satisfy his need for self-presentation and validation (cf. Brailovskaia \& Bierhoff, 2012; 2016; Mehdizadeh, 2011; Ozimek et al., 2018), and a person scoring high on materialism may use Facebook as means for acquiring so-called "Facebook friends" as digital possession (cf. Chu, Windels, \& Kamal, 2016; Kamal, Chu, \& Pedram, 2013; Ozimek et al., 2017; Ozimek \& Förster, 2017). Thus, the activated goals differ with respect to users' needs and personality. However, self-regulation theories suggest that the final goal of self-regulation (what we call "the main motive") is happiness (cf., Carver \& Scheier, 1973; Kruglanski et al., 2002). Facebook use may serve this goal besides other goals (e.g., enjoyment, distraction, and building social networks). Nevertheless, regardless of whether one uses Facebook for social comparison, self-presentation, or acquiring possession, Facebook, as well as other social media, is frequently used as means for reaching happiness (cf., Ozimek et al., 2017). However, based on the SOS-T's proposition that social comparison works as a motive which activates certain goals and, depending on persons' social comparison orientation, they either use social media as a means to their personal ends or they do not (see Ozimek et al., 2017), it can be hypothesized that a high social comparison orientation is associated with higher social Facebook use (i.e., a higher intensity and more time spend on Facebook) and that this association can be partly explained through the striving for personal goals, such as positive comparison with other people, whereby Facebook is used as a means for.

\section{Hypotheses}

In correspondence with former studies, high social comparison orientation should be associated with high Facebook usage:

- H1: Social comparison orientation is positively correlated with intensity of social Facebook use.
In correspondence with previous findings, difficulties in emotion regulation should be associated with higher intensity of social Facebook use. This hypothesis is derived from the results of a study by Hormes, Kearns, and Timko (2014) on online social network use. Specifically, the results indicate that low skills in emotion regulation are associated with dysfunctional online social network use leading to heavy use of Facebook or even to Facebook addiction. Therefore, we propose the following hypothesis:

- H2: Difficulties in emotion regulation are positively correlated with intensity of social Facebook use.

As self-regulation mainly works unconsciously (Förster \& Jostmann, 2015; see also Gross, 1998; Kruglanski et al., 2002), it can be primed. We employed priming that reminded participants of their own unachieved goals. Specifically, reminding participants of their unachieved goals is likely to pre-activate such goals. Note that former research on the priming of goals have demonstrated on the basis of six studies that not yet realized goals are easily primed and that they activate related action tendencies (Aarts, Gollwitzer \& Hassin, 2004). This principle, which was labelled as goal contagion, is summarized by the proposition: "Perceiving is for pursuing" (p. 23). The effectiveness of goal priming was, for example, shown with respect to self-regulation of eating (Papies \& Hamstra, 2010). It is not limited to the content, which was explicitly primed, but also generalizes to broader categories (e.g., money: agreement with free-market capitalism; cf. Caruso, Baxter, Vohs \& Waytz, 2013). Besides goal contagion, another mechanism, which focuses on the comparison between the actual self and the ideal self is likely to activate corresponding action tendencies after priming: The preactivation of unfulfilled goals is likely to induce participants to socially compare themselves in order to reinstate a positive self-view. Taken together, both mechanisms should increase participants' need to use Facebook (socially) in order to reach positive emotional end states, as the SOS-T predicts. Thus, the pre-activation of unachieved goals should increase participants' need to regulate their self by the means of using Facebook.

- H3a: Priming of unachieved goals is related to higher intensity of social Facebook use.

Because difficulties in emotion regulation reduce the level of self-control, elicit impairments of concentration, and reduce the number of available methods of emotion regulation, such difficulties should moderate the priming effect with higher difficulties producing a stronger priming effect (compared with individuals who exhibit lower difficulties in emotion regulation). Therefore, the hypothesis is plausible that: 
- H3b: Higher difficulties in emotion regulation lead to a stronger priming effect of unachieved goals with respect to intensity of social Facebook use.

This hypothesis is summarized as follows with IV standing for independent variable, MOD for moderator, and DV for dependent variable:

Priming of unachieved goals (IV) $\rightarrow$ difficulties in emotion regulation (MOD) $\rightarrow$ intensity of social Facebook use (DV).

Specifically, participants who tend to show stronger emotional disturbances in emotionally arousing situations are likely to favour global offers of goal attainment. Facebook is promising in this respect because it is not set on one or the other strategy and participants do not have to scrutinize in advance what the best strategy would be to find out more about their goals. At the same time, the use of Facebook promises to learn something about own goals. Such a global promise should be highly attractive for people who are frequently caught in emotionally disturbing thoughts. From this reasoning, we derive the assumption that difficulties in emotion regulation moderate the relationship between priming of unachieved goals and need for using Facebook.

A high social comparison orientation should intensify the priming effect as well because especially Facebook offers an ideal possibility of social comparisons in order to clarify one's own progress toward goal attainment (compared with others). Social comparison serves the function to find out where the person who performs the comparison stands on social dimensions. The sequence of self-regulation in the face of activated goals in case of high social comparison orientation is likely to be: My level of goal attainment may be derived from comparisons with relevant others which are possible on Facebook. In contrast, the priming of goals in the case of low social comparison orientation is likely to be more focused on the identification of own steps toward goal attainment which is not dependent on comparisons with others.

- H3c: High social comparison orientation leads to a stronger priming effect of unachieved goals with respect to intensity of social Facebook use [Priming of unachieved goals (IV) $\rightarrow$ social comparison orientation (MOD) $\rightarrow$ intensity of social Facebook use (DV)]

\section{Method}

An online survey was constructed with the Questback software Unipark in order to examine the aforementioned hypotheses. Participants were recruited via an online portal of the Ruhr University of Bochum, social networks such as Facebook itself, as well as posters and personal contact. The inclusion criterion was the usage of a private Facebook account. The survey was held in German language and was meant to be answered in 15 to $20 \mathrm{~min}$.

Participants were informed about the background of the study, as well as the fact that participation was voluntary. They were instructed to thoroughly read the questions and answer them spontaneously but conscientiously, even though some of them might sound similar. It was stated that their data would be handled confidentially and only used for the purpose of the study. No personal conclusions could be drawn from their answers.

\section{Overview}

First, the participants responded to several demographic questions. Specifically, they were asked to state their age, gender, relationship status, highest education, and their current occupation. Furthermore, it was inquired whether they fulfilled the inclusion criterion. If this was not the case, the participants were led to an end page which thanked them for their participation. The number of Facebook friends was asked for as well and could be answered by moving a slider bar on a scale (< 50, 50-100, 100-200, 200-300, 300-400, 400-500, > $500)$. It was then asked with what percentage of these friends the participant communicated exclusively on Facebook.

\section{Measures}

Difficulties in emotion regulation are measured by the Difficulties With Emotion Regulation Scale (DERS; Gratz \& Roemer, 2004) which was used in the German version by Führer and Rohmann (in preparation). The DERS measures deficits in emotional regulation either because of insufficient modulation of emotional arousal, because of ignorance of own emotions, or because of restricted ability to act in desired ways while emotionally aroused. The opposite of high difficulties in emotion regulation is to be clear about one's own feelings and to be able to get things done even if emotional arousal occurs (Gratz \& Roemer, 2004). Participants are asked to specify how often 36 statements such as "When I'm upset, I feel guilty for feeling that way" or "I have no idea how I am feeling" apply to them (response range from $1=$ almost never $(0-10 \%)$ to $5=$ almost always (91-100\%). Thus, higher ratings indicate higher difficulties with emotion regulation. The DERS contains six reliable subscales with respect to difficulties in emotion regulation of: Not Acceptance (1), Goals (2), Impulse (3), Awareness (4), Strategy (5) and Clarity (6). Former studies have shown that the calculation of an overall index $\left(\mathrm{DERS}_{\text {Total }}\right)$ is viable because the subscales are highly correlated with each other (cf. Führer \& Rohmann, in prep.; for correlations and internal consistencies see also Appendix Table 7). Therefore, the subscales were not further analysed.

We constructed an overall index of difficulties in emotion regulation by averaging across items. In correspondence with 
the American scale (Gratz \& Roemer, 2004) and a Turkish version of the DERS (Santas-Atalar, Gencöz \& Özen, (2015) the German version of the DERS turned out to be internally consistent, $\alpha=.94)$.

For measuring social comparison orientation, the Iowa Netherlands Comparison Orientation Measure (INCOM; Gibbons \& Buunk, 1999) was used in the German version by Schneider and Schupp (2011), which shows good reliability and validity. Schneider and Schupp found internal consistencies of $\alpha=0.7-0.85$ for a German population. The questionnaire is composed of eleven statements such as "I often compare how I am doing socially (e.g., social skills, popularity) with other people" or "If I want to learn more about something, I try to find out what others think about it". The response scale is a 5-point Likert scale (ranging from $1=I$ disagree strongly to $5=$ I agree strongly). Higher ratings indicate a higher social comparison orientation. The current internal consistency is $\alpha=.84$.

Intensity of social Facebook use was measured with the Facebook Activity Questionnaire (FAQ) by McAndrew and Jeong (2012) in the German version by Ozimek and Bierhoff (2016). It consists of 30 items, based on the three factors Watching (11 items, e.g. 'I'm looking at others' relationship status”), Impressing (6 items, e.g. „I'm struggling to decide which profile picture I would like to post'), and Acting (13 items, e.g. ,I'm posting photographs”).

Based on the German version for all items, frequency assessments were registered. The response scale ranged from never (1) to very often (5), thus higher ratings indicate higher Facebook use. Ozimek and Bierhoff (2016) found good internal consistency for the three dimensions ( $\alpha_{\text {watching }}=.83$, $\left.\alpha_{\text {impressing }}=.79, \alpha_{\text {acting }}=.77\right)$ and the overall questionnaire FAQ $Q_{\text {Total }}(\alpha=.87)$. Current internal consistencies are $\alpha_{\text {watching }}=.86, \alpha_{\text {impressing }}=.80, \alpha_{\text {acting }}=.81$ and the overall questionnaire $F A Q_{\text {Total }}(\alpha=.89)$. Former studies have shown that the calculation of an overall index $\left(\mathrm{FAQ}_{\mathrm{Total}}\right)$ is viable because the subscales are highly correlated with each other (cf. Brandenberg, Ozimek, Bierhoff, \& Janker, 2019; Ozimek \& Bierhoff, 2016). Therefore, the subscales were not further analysed.

Whereas the FAQ measures actual Facebook usage directly, it measures participants' future Facebook use indirectly because $F A Q_{\text {Total }}$ is highly correlated with the answer to the question "How likely would you like to log in on Facebook right now?" (Ozimek, Förster, \& Blaschke, in prep.; Ozimek, Hanke, Führer, \& Förster, in prep; cited by Ozimek \& Förster, 2017). Therefore, the FAQ assesses explicitly the level of Facebook activity and implicitly the need for using Facebook socially.

Life satisfaction was included as a control variable. It was measured with the German version of the Satisfaction With Life Scale (SWLS, Diener, Emmons, Larsen, \& Griffin, 1985; translated by Schumacher, 2003). A total score is calculated as the average across the five items of the SWLS (e.g., "In most ways my life is close to my ideal"), each of which are answered on a 7-point Likert scale from $1=$ strongly disagree to $7=$ strongly agree. Thus, higher ratings indicate a higher life satisfaction. The current internal consistency of the SWLS is $\alpha=.86$.

\section{Experimental Design}

The occurrence of priming effects is presumably unconscious because the participants are unaware of the relation between the priming task and the dependent measures (Förster \& Jostmann, 2015). The priming of unaccomplished goals is meant to activate intentions to attain them (cf., Aarts, Gollwitzer \& Hassin, 2004). By reminding the participants of their unaccomplished life goals, participants are forced to unconsciously compare the person they want to be, their ideal self, with the person they are at the moment, their actual self. This comparison presumably evokes intentions to realize some of their goals, given the need to self-regulate, which, among other options, can be satisfied by socially comparing themselves to others in order to evaluate their action alternatives. As Facebook offers the nearly ideal platform for performing social comparisons, primed participants were expected to realize their intention to attain their goals by consulting information about friends and other persons on Facebook.

Participants were randomly assigned to priming conditions. Three priming groups were created: priming of materialistic goals $(n=110)$, priming of non-materialistic goals $(n=122)$, and no priming control group $(n=109)$. However, no significant differences emerged depending on materialistic vs. nonmaterialistic goals. Therefore, both priming subgroups were collapsed $(n=232)$. The following priming instructions were employed: In the priming of materialistic goals condition participants read a short text defining materialistic goals. It was mentioned that people have different goals in life. Some of them they already achieved, others they have not achieved yet. Materialistic goals are defined as goals including materialistic issues, for example 'owning a car' or 'being rich'. The instruction was continued as follows: 'Please take two minutes now and write down all the materialistic goals that you personally still want to achieve.' In the priming of non-materialistic goals condition most of the instruction was the same as in the materialistic goal condition. The only difference was that nonmaterialistic goals were mentioned defined as goals including non-materialistic issues, for example 'to finish your studies' or 'to start a family'. In the no priming control condition participants had to write down the names of as many animals starting with the letter B as they could think of in two minutes. This task was chosen because it was completely independent of goal setting and emotionally neutral. In all conditions it was up to the participants whether they wanted to use a stop watch or look at the time; the two minutes limit was given in order to 
make the different group conditions comparable, as well as to standardize the priming procedure. ${ }^{1}$ Following the priming manipulation participants' intensity of social Facebook use was measured with the FAQ.

\section{Participants}

The data exported from Unipark included 355 participants in the beginning. After deleting the cases which did not fulfil the inclusion criterion, the data sample encompassed 341 cases. $72.2 \%$ (248) participants stated female gender, $26.1 \%$ (89) were male and $1.2 \%$ (4) identified as neither female nor male. Participants' age ranged from 15 to 69 years, with a mean of $M=28.79$ years $(S D=9.43)$. Many participants had a high-school diploma (Abitur) and/or a college/university degree (41.3\% resp. $44.9 \%)$. In terms of current occupation, 66 (19.4\%) stated to be studying psychology and 138 (40.5\%) were studying a different field. 110 participants $(32.3 \%)$ were employed and the rest was either in training $(2.1 \%)$, unemployed $(1.5 \%)$, home keeper $(1.2 \%)$, retired $(1.2 \%)$ or "other" $(2.1 \%)$. The sample consisted of 119 singles $(34.9 \%)$ and 172 persons in a relationship (50.4\%), and 50 other participants were married (14.7\%). By using the randomized trigger for grouping participants, they were distributed to the two groups.

\section{Results}

\section{Preliminary Analysis}

The statistical analysis was conducted with the program IBM SPSS Statistics 25. Preliminary analysis showed no effects of condition on demographics (see Table 1). This result confirms the effectiveness of the random assignment. The distribution of dependent and control variables was tested for approximation to a normal distribution both by visual assessment and by considering skewness and kurtosis. No significant deviations

\footnotetext{
${ }^{1}$ Because priming effects vanish rapidly (cf. Förster \& Liberman, 2007), we did not include a manipulation check on the effectiveness of the priming. But many published (e.g., Aarts, Gollwitzer \& Hassin, 2004) and unpublished studies show that the awareness of unachieved goals is associated with higher self-regulative processes (cf. Ozimek, P., Förster, J., \& Blaschke, L., (in prepa). Millions of users, millions of social comparisons? - the impact of Facebookexposure on social comparison orientation: A priming study; Ozimek, P., Hanke, S., Führer, A. \& Förster, J. (in prep-b). The impact of self-esteem on social Facebook use: A priming study cited by Ozimek et al., 2017). Another problem with including a manipulation check is that it might elicit suspicion with respect to the priming. Note that priming effects vanish when the predicted effect can be anticipated by participants (cf. Förster \& Jostmann, 2015, Förster \& Liberman, 2007). In addition, the priming manipulation of unachieved goals represents high content validity because the instruction focuses on unachieved goals by saying 'write down all the materialistic/nonmaterialistic goals that you personally still want to achieve'. Its high content validity permits to interpret the priming instruction as a pre-activation of personal goals (in comparison with the control condition which focuses on listing names of animals).
}

Table 1 Demographic differences between priming groups

\begin{tabular}{lll}
\hline & $\chi^{2} / \mathrm{t}(\mathrm{df})$ & $p$ \\
\hline Gender & $1.88(2)$ & .391 \\
Highest education & $2.81(6)$ & .886 \\
Current occupation & $8.36(7)$ & .299 \\
Relationship status & $1.03(2)$ & .597 \\
Age & $.11(339)$ & .915 \\
FB friends & $.04(339)$ & .970 \\
$\%$ FB only-friends & $-.47(339)$ & .636 \\
\hline
\end{tabular}

Note. FB friends = the estimated number of friends the person has on Facebook. \% FB only-friends $=$ the percentage of those friends that the participant is only connected with via Facebook, not in real life

from the normal distribution were found with respect to DERS, INCOM, FAQ, and SWLS. In addition, scale means as a function of priming are displayed in Table 2. As expected on the basis of the random assignment, no significant differences emerged between the priming conditions on DERS, INCOM, and SWLS. Additionally, you will find a table with all means, derivations and group comparisons with respect to the subscales in Appendix Table 6 .

\section{Testing the Hypotheses}

Pearson correlations were calculated between FAQ, DERS and INCOM. These correlations (including $S W L S$ ) are summarized in Table $3 .^{2}$ In general, significant correlations were found between all scales included, apart from the correlation of Satisfaction with Life with intensity of social Facebook use. According to Cohen (1988), a correlation of $r=.10$ is small, $r=.30$ is medium and $r=.50$ is high. The correlations summarized in Table 3 fall in the range of small to medium magnitude. The negative correlation between SWLS and DERS confirms the construct validity of the DERS because higher difficulties in emotion regulation should be associated with less life satisfaction. Intensity of social Facebook use and Social Comparison Orientation were positively related confirming H1. In addition, intensity of social Facebook use and Difficulties with Emotion Regulation were also correlated positively. This significant correlation supports $\mathrm{H} 2$.

The priming of unaccomplished goals was assumed to lead to a higher intensity of social Facebook use (H3a). This hypothesis was tested by comparing the mean $F A Q$ scores between the priming and control group (see Table 2). Because the $t$-test was not significant, H3a was rejected. Comparison of the means of the FAQ subscales Watching $(t(337)=1.04, p=.30)$, Impressing $(t(337)=-.03, p=.98)$, and Acting $(t(333)=-.22$, $p=.83$ ) revealed the same nonsignificant result (cf. Appendix Table 6).

\footnotetext{
$\overline{{ }^{2} \text { You will find }}$ a full correlation matrix with all subscales in Appendix Table 7.
} 
Table 2 Scale means as a function of priming

\begin{tabular}{|c|c|c|c|c|c|c|c|c|}
\hline & \multicolumn{2}{|c|}{ Control $(n=109)$} & \multicolumn{2}{|c|}{ Priming $(n=232)$} & \multirow[b]{2}{*}{$t(d f)$} & \multirow[b]{2}{*}{$p$} & \multicolumn{2}{|c|}{ Total $(N=341)$} \\
\hline & $M$ & $S D$ & $M$ & $S D$ & & & $M$ & $S D$ \\
\hline DERS & 2.48 & .62 & 2.43 & .58 & $.67(325)$ & .471 & 2.45 & .59 \\
\hline INCOM & 3.37 & .67 & 3.20 & .70 & $2.07(333)$ & .382 & 3.25 & .70 \\
\hline FAQ & 2.58 & .47 & 2.56 & .52 & $.30(330)$ & .762 & 2.56 & .50 \\
\hline SWLS & 4.92 & 1.10 & 5.00 & 1.16 & $-.531(335)$ & .596 & 4.98 & 1.13 \\
\hline
\end{tabular}

Note . DERS $=$ Difficulties With Emotion Regulation, INCOM = Social Comparison Orientation, FAQ = Intensity of social Facebook use, SWLS = Satisfaction with Life Scale. $\mathrm{M}=$ Mean, $\mathrm{SD}=$ Standard deviation, $\mathrm{df}=$ degrees of freedom

In order to test the hypothesis that more difficulties in emotion regulation will lead to a stronger effect of the priming on intensity of social Facebook use (H3b), the relevant variables were standardized before a moderation analysis was conducted using the PROCESS macro for SPSS (Hayes, 2013), version 3.2. In advance, the model requirements of homoscedasticity as well as independent and normally distributed residuals were tested. All criteria were met for performing a multiple regression analysis.

The moderation model with Difficulties with Emotion Regulation and priming estimating intensity of social Facebook use was significant, $R^{2}=.044, F(3,316)=$ $7.79, p=.003$. The interaction term accounted for a significant proportion of the variance in FAQ, $\Delta R^{2}=.0142$, $F(1,316)=4.695, p=.031$. Upon examination of the interaction plot, it is visible that for individuals with a lower DERS score, the priming leads to a lower intensity of social Facebook use, compared to the control group. On the other hand, for participants with a higher DERS score the priming led to higher intensity of social Facebook use (see Fig. 1). The statistical results of the moderation analysis are summarized in Table 4.

In $\mathrm{H} 3 \mathrm{c}$ we assumed that high Social Comparison Orientation enhances the priming effect. This hypothesis was examined by a second moderation model which was calculated with Social Comparison Orientation and priming estimating intensity of social Facebook use after standardizing variables. The model was highly

Table 3 Correlations of intensity of Facebook use, Difficulties with Emotion Regulation, Social Comparison Orientation and Satisfaction with Life Scale

\begin{tabular}{llll}
\hline & 1 & 2 & 3 \\
\hline FAQ (1) & - & & \\
DERS (2) & $.180^{* *}$ & - & \\
INCOM (3) & $.255^{* *}$ & $.327^{* *}$ & - \\
SWLS & -.014 & $-.439 * *$ & $-.123 *$ \\
\hline
\end{tabular}

Note. $\mathrm{FAQ}=$ Intensity of social Facebook use, DERS = Difficulties with Emotion Regulation, INCOM = Social Comparison Orientation, SWLS $=$ Satisfaction with Life Scale. $* p<.05 * * p<.001$ significant, $R^{2}=.069, F(3,322)=8.00, p<.001$, as was the main effect of social comparison orientation. This time, however, the interaction term did not become significant (see Table 5).

Therefore, H3c was not confirmed. The correspondent graph in fig. 2, shows a tendency of participants with high Social Comparison Orientation who have less of intensity of social Facebook use when in the priming group, and people with low Social Comparison Orientation wanting to use Facebook more socially when in the priming group. It is also visible that there is already a big difference in intensity of social Facebook use between the low and high social comparers, which corresponds with the positive correlation between both variables (cf., Table 3) supporting H1.

For both moderations, several control variables were considered in order to check if they changed the results. This was not the case, however. Neither gender, age, participants' education, current occupation, relationship status, number of Facebook friends, nor life satisfaction had a significant impact on the moderation effects.

\section{Discussion}

$\mathrm{H} 1$ and $\mathrm{H} 2$ covered the association between Facebook usage and social comparison orientation as well as difficulties in emotion regulation. With respect to $\mathrm{H} 1$ which was confirmed by the correlational results several studies have already shown a positive association between social comparison orientation and intensity of social Facebook use. Therefore, the empirical evidence for $\mathrm{H} 1$ is substantial although it is mainly based on correlational data. The hypothesis is based on the following explanation: The many opportunities Facebook offers for people to present and compare themselves to and with others attracts people who focus on social comparisons (= high social comparison orientation). These people presumably show a higher intensity of social Facebook use because they like to perform social comparisons which are offered in abundance on Facebook (cf., Appel et al., 2016; Lee, 2014; Ozimek et al., 
Fig. 1 Conditional effects of priming on intensity of social Facebook use among those relatively low and relatively high in their Difficulties with Emotion Regulation

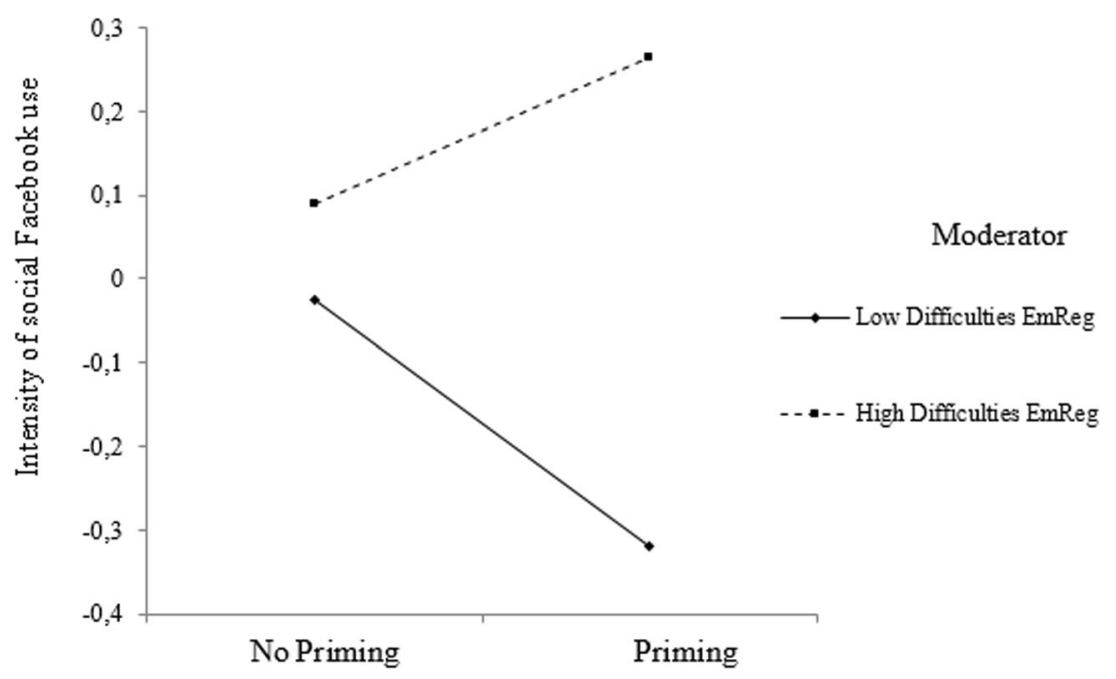

2017; Ozimek \& Förster, 2017; Ozimek \& Bierhoff, 2019; Verduyn et al., 2017). This is also in line with the SOS-T (Ozimek \& Förster, 2017) proposing that people use Facebook as means to reach personal goals and motives such as satisfying their need for social comparison (cf. Ozimek \& Bierhoff, 2016; Ozimek \& Förster, 2017).

In correspondence with $\mathrm{H} 2$, people with high difficulties in emotion regulation showed a higher Facebook usage. The hypothesis was derived from results reported by Hormes et al. (2014) showing that poor emotion regulation skills could lead to dysfunctional and even disordered online social network use. As our test of $\mathrm{H} 2$ is based on correlational design, it is unclear whether people with more problems in emotion regulation have a general tendency to use Facebook more, or whether intense Facebook usage leads to problems in emotion regulation. Only experimental or longitudinal data might clarify which interpretation of the data is viable.

Hypothesis $3 \mathrm{a}$ assumed that priming of unaccomplished goals would lead to a higher intensity of social Facebook use. The nonsignificant result of the comparison between the priming conditions refuted $\mathrm{H} 3 \mathrm{a}$. Priming unaccomplished goals did not increase participants' intensity of social
Facebook use. The means of FAQ in the two priming conditions were nearly equal.

Why was the priming of goals not successful in terms of intensity of social Facebook use? As proposed by the $S O S-T$, people use Facebook in order to reach positive emotional states, so it was assumed that they would want to access it even more after the priming of goals. This derivation from SOS-T was clearly rejected by the data. However, this conclusion ignores the occurrence of a person-situation interaction which indicated that in correspondence with $\mathrm{H} 3 \mathrm{~b}$ the effect of priming unaccomplished goals was moderated by difficulties in emotion regulation: The higher the difficulties in emotion regulation, the more the participants' need to use Facebook socially. Although H3a was rejected overall, it is nevertheless viable for people who exhibit high difficulties in emotion regulation. Note that the occurrence of person-situation interactions is in line with Lewin's field theory (1935; see also Ross \& Nisbett, 1991). In the current study, the person is represented by the difficulties in emotion regulation whereas the situation is created by the priming of unaccomplished goals. Furthermore, person-
Table 4 Results from moderation model with Difficulties with Emotion Regulation and priming estimating intensity of social Facebook use

\begin{tabular}{lllll}
\hline & $b$ & $S E B$ & $t$ & $p$ \\
\hline Constant & $.002[-.106, .110]$ & .054 & .040 & .968 \\
Priming group (IV) & $-.030[-.139, .079]$ & .055 & -.545 & .586 \\
DERS (MOD) & $.175[.066, .284]$ & .055 & 3.150 & .002 \\
Interaction & $.118[.011, .225]$ & .055 & 2.170 & .031 \\
(IV x MOD) & & & &
\end{tabular}

Note. $R^{2}=.044 ;$ IV = Independent variable, $\mathrm{MOD}=$ Moderator, DERS $=$ Difficulties in Emotion Regulation Scale
Table 5 Results from moderation model with Social Comparison Orientation and priming estimating intensity of social Facebook use

\begin{tabular}{lllll}
\hline & $b$ & $S E B$ & $t$ & $p$ \\
\hline Constant & $.004[-.102, .110]$ & .054 & .066 & .947 \\
Priming group (IV) & $.013[-.093, .120]$ & .054 & .241 & .810 \\
SCO (MOD) & $.260[.154, .365]$ & .054 & 4.821 & $<.001$ \\
Interaction & $.035[-.072, .143]$ & .055 & .658 & .520 \\
(IV X MOD) & & & & \\
\hline
\end{tabular}

Note. $R^{2}=.07 ; \mathrm{IV}=$ Independent variable, $\mathrm{MOD}=$ Moderator, $\mathrm{SCO}=$ Social Comparison Orientation 
Fig. 2 Conditional effects of priming on intensity of social Facebook use among those relatively low and relatively high in their Social Comparison Orientation

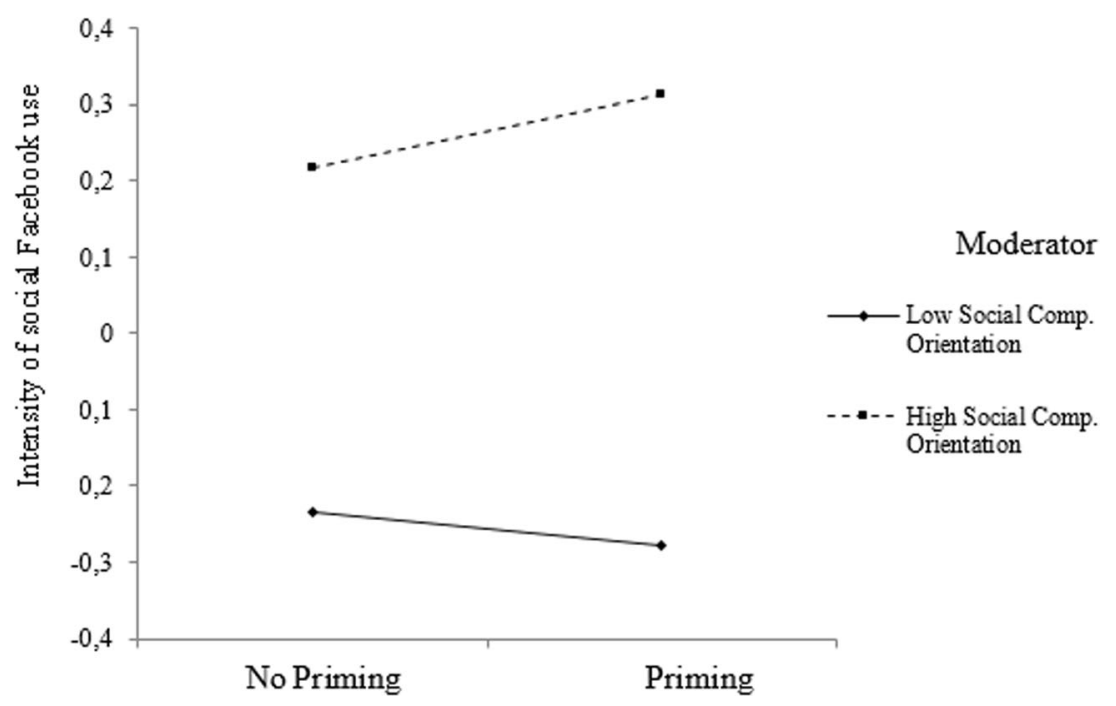

situation effects in priming research are quite common (e.g., Ozimek \& Förster, 2017). They are congruent with psychological studies assuming that there is not one but several factors determining behaviour, those factors usually being of both situational and biological nature. In medicine this principle is incorporated in the biopsychosocial model by Engel (1977).

In the derivation of $\mathrm{H} 3 \mathrm{~b}$ we had assumed that difficulties with emotional regulation are likely to impair self-regulation and to reduce the number of available options of emotional regulation. Therefore, it was assumed that difficulties in emotion regulation moderate the priming effect of goals by leading to stronger priming effects among people who exhibit higher difficulties with emotion regulation (compared with people who exhibit less difficulties with emotional regulation). The argument was fully elaborated in the introduction. Suffice it to say that difficulties with emotion regulation refer to a central component of lack of self-regulation and, therefore, are a very plausible moderator of the priming effect of goals which was supposed to activate self-regulation. This is in line with the Social Compensation Hypothesis ("poor get richer") implying that offline unpopularity is tried to be compensated online (cf., Valkenburg, Schouten, \& Peter, 2005) as well as the SOS-T (Ozimek \& Förster, 2017) proposing that people use SNSs for self-regulation. Thus, being reminded on unfulfilled personal goals people seem to use SNS such as Facebook as means to reach their goals and to regulate themselves, especially when they do have high difficulties in emotion regulation. H3c, concerning social comparison orientation as moderator was rejected, because there were no significant differences of the priming effect between persons with low or high social comparison orientation. There was, however, a trend towards the expected result of a higher social comparison orientation leading to a stronger need to use Facebook in the priming group. This was in line with the theoretical assumption which emphasized the relevance of social comparison needs in intensity of social Facebook use (cf. Ozimek \& Förster, 2017). For people with a higher social comparison orientation, the priming effect of goals was thought to be especially strong (cf. the reasoning with respect to $\mathrm{H} 3 \mathrm{c}$ in the introduction). But further interpretations are not warranted because no significant moderation effect including social comparison orientation emerged. Several control variables were included in the moderation model but did not cause any significant change of the primary results.

With respect to results by Gibbons \& Gerrard (1989) on differences in mood improvement between participants with low and high self-esteem, depending on whether the social comparison was upward or downward, it seems possible that participants' self-esteem could be an important factor which should be examined in the future, as well as the question of culture-specific results because social comparison orientation was found to differ considerably between cultures (Guimond et al., 2007).

Another promising individual difference variable which should be considered in future research on goal priming is optimism-pessimism (cf. Rohmann \& Bierhoff 2016) because the pre-activation of goals should result in different emotional states depending on optimism (optimistic people should respond with more positive affect) and pessimism (pessimistic people should respond with more negative affect). General expectancies of consequences (i.e., dispositional optimism) is measured by the Life Orientation Test (Scheier, Carver \& Bridges, 1994). In general, the affective consequences of goal priming are likely to depend on the level of dispositional optimism. As predicted by the SOS-T, people use Facebook in order to reach positive emotional states. This need should be stronger for pessimistic than optimistic people. More 
specifically, the priming combined with pessimism is likely to elicit negative feelings in participants. ${ }^{3}$ These negative feelings need to be regulated in order to reach positive emotional states again. Therefore, among people who use Facebook socially priming of goals and dispositional pessimism are assumed to interact with each other. Future research should examine the correctness of this prediction which is based on the assumption of a person-situation interaction.

\section{Limitations}

Online surveys could be seen as preventing the researchers from being able to control the situation completely. Therefore, the results of online surveys need to be interpreted with caution. As a countermeasure, the participants were given a contact address to ask questions if needed. Therefore, the likelihood of misunderstanding the questionnaires was reduced. Moreover, online surveys have benefits as well. They theoretically allow participants from all over the world to participate, if they speak the language the survey is constructed in, making the sample potentially much bigger, more diverse, and more representative than a sample of participants who are, for example, recruited at one university (cf., Zerback, Schoen, Jackob, \& Schlereth, 2009). It would have been possible to further explore the participants' motivations by asking them after the priming whether they were disturbed, distracted or whether they had difficulties with the task. However, as priming effects are thought to fade quickly (Förster \& Liberman, 2007), this was not done in the current study.

Although some of the analyses in this study were correlational, meaning that no causal conclusions can be drawn the effect of the priming on the Facebook Activity Questionnaire can be interpreted in a causal way. However, it is unclear which feelings and thoughts exactly the priming evoked or whether there are other factors which influenced intensity of social Facebook use. Future studies should try to elicit priming effects on the basis of the pre-activation of goals in different settings as well as examine alternative theoretical perspectives consistent with the present results.

Finally, Facebook is only one of many SNSs, albeit it represents a highly popular online social network site. It has yet to be determined whether the results, including those on moderation by difficulties in self-regulation, are replicable on the basis of other SNSs. As an encouragement, there have been some recent findings based on the professional social network XING

\footnotetext{
${ }^{3}$ A single priming study is not able to test both potential outcomes as well as underlying mechanism. For this, we do not claim to present an exhaustive network of variables which might influence goal priming effects. Instead we point out first insights in the interplay between intensity of social Facebook use and self-regulation. Underlying mechanisms, such as negative emotions caused by the pre-activation of unachieved goals in pessimistic people have to be examined in future studies.
}

(Brandenberg et al., 2019; Ozimek \& Bierhoff, 2019) which show that the results might be replicable across different SNSs.

The present study focused on intensity of social Facebook use. Note that Facebook does not focus on social exchange exclusively, but other social networks do, such as messaging apps like WhatsApp, or other social networking sites such as twitter or reddit. In summary, the results of this study cannot be generalized to other SNSs by now and further research is needed on the issue of generalizability of the results.

\section{Conclusion}

The aim of this study was to further explore the Social Online Self-Regulation Theory and its assumptions, especially with respect to emotion regulation and social comparison orientation, in order to better understand Facebook users' social behaviour. In overview, the results, seem to indicate that self-regulation can indeed be a reason for people who want to use Facebook socially, but only depending on individual differences in difficulties with emotion regulation.

Such difficulties could be a possible risk factor for eliciting a Facebook addiction disorder ( $F A D$; Fenichel, 2009; Brailovskaia, Rohmann, Bierhoff, \& Margraf 2018). Similar results were reported by Hormes et al. (2014), who concluded that difficulties with emotion regulation are associated with dysfunctional Facebook usage as well as vulnerability to Facebook addiction. Since $F A D$ is still not an officially recognised disorder in the Diagnostic and Statistical Manual of Mental Disorders (5th ed., DSM-5; American Psychiatric Association, 2013), it is important to understand its development and maintenance and eventually create effective intervention programs (Brailovskaia \& Margraf, 2017). The study at hand might be another step in that direction, but more evidence on the limits of negative repercussions on the individual remain to be established.

Not only in the light of $F A D$ are the findings of this study relevant for current and future research. In addition, they support the $S O S-T$ extended on the prediction of person-situation interactions. The SOS-T could provide a fundamental understanding of the motives behind the use of Facebook and has recently been supported by the results of several other studies (Brandenberg et al., 2019; Ozimek et al., 2017; Ozimek \& Förster, 2017; Ozimek et al., 2018; Ozimek \& Bierhoff, 2019).

Funding Information Open Access funding provided by Projekt DEAL.

Compliance with Ethical Standards We declare that our study was approved by the local ethical committee of the Ruhr University of Bochum, department of psychology.

Conflict of Interest none. 
Declarations We declare that there are no potential conflicts of interests, and that informed consent was given by all participants.

\section{APPENDIX}

Table 6 Scale and subscale means as a function of priming

\begin{tabular}{|c|c|c|c|c|c|c|c|c|}
\hline & \multicolumn{2}{|c|}{ Control $(n=109)$} & \multicolumn{2}{|c|}{ Priming $(n=232)$} & \multirow[b]{2}{*}{$t(d f)$} & \multirow[b]{2}{*}{$p$} & \multicolumn{2}{|c|}{ Total $(N=341)$} \\
\hline & $M$ & $S D$ & $M$ & $S D$ & & & $M$ & $S D$ \\
\hline DERS_Total $(\alpha=.94)$ & 2.48 & .62 & 2.43 & .58 & $.67(325)$ & .471 & 2.45 & .59 \\
\hline DERS_NA $(\alpha=.87)$ & 2.64 & .96 & 2.58 & .88 & $-.52(337)$ & .601 & 2.60 & .91 \\
\hline DERS_G $(\alpha=.90)$ & 3.12 & .91 & 3.01 & .86 & $-1.08(337)$ & .282 & 3.05 & .88 \\
\hline DERS_I $(\alpha=.85)$ & 2.34 & .83 & 2.27 & .77 & $-.70(336)$ & .486 & 2.30 & .79 \\
\hline DERS_A $(\alpha=.74)$ & 2.32 & .56 & 2.30 & .59 & $-.23(334)$ & .818 & 2.31 & .60 \\
\hline DERS_S $(\alpha=.88)$ & 2.49 & .83 & 2.44 & .80 & $-.55(338)$ & .585 & 2.46 & .81 \\
\hline DERS_C $(\alpha=.87)$ & 2.20 & .74 & 2.18 & .71 & $-.17(338)$ & .862 & 2.19 & .72 \\
\hline INCOM_Total $(\alpha=.84)$ & 3.37 & .67 & 3.20 & .70 & $2.07(333)$ & .382 & 3.25 & .70 \\
\hline FAQ_Total $(\alpha=.89)$ & 2.58 & .47 & 2.56 & .52 & $.30(330)$ & .762 & 2.56 & .50 \\
\hline FAQ_W $(\alpha=.86)$ & 2.51 & .62 & 2.43 & .65 & $-1.04(337)$ & .301 & 2.46 & .64 \\
\hline FAQ_I $(\alpha=.80)$ & 3.68 & .81 & 3.68 & .79 & $.030(337)$ & .976 & 3.68 & .80 \\
\hline FAQ_A $(\alpha=.81)$ & 2.14 & .51 & 2.15 & .55 & $.219(337)$ & .827 & 2.14 & .54 \\
\hline $\operatorname{SWLS}(\alpha=.86)$ & 4.92 & 1.10 & 5.00 & 1.16 & $-.531(335)$ & .596 & 4.98 & 1.13 \\
\hline
\end{tabular}

Note . DERS $=$ Difficulties with Emotion Regulation, NA = not accepting, $\mathrm{G}=$ goals, $\mathrm{I}=$ impulse, $\mathrm{A}=$ awareness, $\mathrm{S}=$ strategy, $\mathrm{C}=$ clarity, INCOM = Social Comparison Orientation, FAQ = Intensity of social Facebook Use, W = watching, I = impressing, A = acting, SWLS = Satisfaction With Life Scale. $\mathrm{M}=$ Mean, $\mathrm{SD}=$ Standard deviation, $\mathrm{df}=$ degrees of freedom

Table 7 Correlations of Facebook activity, Difficulties with Emotion Regulation, Social Comparison Orientation and Satisfaction with Life Scale with subscales

\begin{tabular}{|c|c|c|c|c|c|c|c|c|c|c|c|c|}
\hline & 1 & 2 & 3 & 4 & 5 & 6 & 7 & 8 & 9 & 10 & 11 & 12 \\
\hline FAQ_Total (1) & - & & & & & & & & & & & \\
\hline FAQ_W (2) & $.808 * * *$ & - & & & & & & & & & & \\
\hline FAQ_I (3) & $.742 * * *$ & $.408 * * *$ & - & & & & & & & & & \\
\hline FAQ_A (4) & $.838 * * *$ & $.458 * * *$ & $.501 * * *$ & - & & & & & & & & \\
\hline DERS_Total (5) & $.180 * *$ & $.147 * *$ & .095 ns. & $.149 * *$ & - & & & & & & & \\
\hline DERS_NA (6) & $.177 * *$ & $.133 *$ & $.149 * *$ & $.150 * *$ & $.793 * * *$ & - & & & & & & \\
\hline DERS_G (7) & $.151 * *$ & $.121 *$ & $.149 * *$ & $.093 \mathrm{~ns}$. & $.693 * * *$ & $.438 * * *$ & - & & & & & \\
\hline DERS_I (8) & $.235 * * *$ & $.201 * *$ & $.112 *$ & $.229 * * *$ & $.843 * * *$ & $.569 * * *$ & $.589 * * *$ & - & & & & \\
\hline DERS_A (9) & $-.050 \mathrm{~ns}$ & $-.023 \mathrm{~ns}$ & $-.104 \mathrm{~ns}$ & $-.013 \mathrm{~ns}$ & $.484 * * *$ & $.188 * *$ & $.078 \mathrm{~ns}$. & $.271 * * *$ & - & & & \\
\hline DERS_S (10) & $.149 * *$ & $.157 * *$ & $.076 \mathrm{~ns}$. & $.117 *$ & $.909 * * *$ & $.731 * * *$ & $.629 * * *$ & $.757 * * *$ & $.296^{* * *}$ & - & & \\
\hline DERS_C (11) & $.080 \mathrm{~ns}$. & $.047 \mathrm{~ns}$ & $.008 \mathrm{~ns}$ & $.117 *$ & $.692 * * *$ & $.473 * * *$ & $.279 * * *$ & $.498 * * *$ & $.504 * * *$ & $.531 * * *$ & - & \\
\hline INCOM (12) & $.255^{* *}$ & $.261 * * *$ & $.258 * * *$ & $.116^{*}$ & $.327 * *$ & $.297 * * *$ & $.341 * * *$ & $.291 * * *$ & $.011 \mathrm{~ns}$ & $.317 * * *$ & $.168 * *$ & - \\
\hline SWLS (13) & -.014 & $-.035 \mathrm{~ns}$ & $.023 \mathrm{~ns}$. & $-.020 \mathrm{~ns}$. & $-.439 * *$ & $-.268 * * *$ & $-.281 * * *$ & $-.330 * * *$ & $-.291 * * *$ & $-.414 * * *$ & $-.387 * * *$ & $-.123 *$ \\
\hline
\end{tabular}

Note. DERS = Difficulties With Emotion Regulation, $N A=$ not accepting, $G=$ goals, $I=$ impulse, $A=$ awareness, $S=$ strategy, $C=$ clarity, INCOM= Social Comparison Orientation, FAQ = Social Facebook Use, $W=$ watching, $I=$ impressing, $A=$ acting, SWLS $=$ Satisfaction With Life Scale. $* \mathrm{p}<.05$ $* * p<.01 * * * \mathrm{p}<.001$ 
Open Access This article is licensed under a Creative Commons Attribution 4.0 International License, which permits use, sharing, adaptation, distribution and reproduction in any medium or format, as long as you give appropriate credit to the original author(s) and the source, provide a link to the Creative Commons licence, and indicate if changes were made. The images or other third party material in this article are included in the article's Creative Commons licence, unless indicated otherwise in a credit line to the material. If material is not included in the article's Creative Commons licence and your intended use is not permitted by statutory regulation or exceeds the permitted use, you will need to obtain permission directly from the copyright holder. To view a copy of this licence, visit http://creativecommons.org/licenses/by/4.0/.

\section{References}

Aarts, H., Gollwitzer,P.M. \& Hassin, R.R. (2004). Goal contagion: Perceiving is for pursuing. Journal of Personality and Social Psychology, 87, 23-37.

American Psychiatric Association. (2013). Diagnostic and statistical manual of mental disorders (5th ed.). DC: Washington.

Appel, H., Gerlach, A. L., \& Crusius, J. (2016). The interplay between social Facebook use, social comparison, envy, and depression. Current Opinion in Psychology, 9, 44- 49.

Bandura, A. (1986). Social foundations of thought and action: A social cognitive theory. Englewood Cliffs: Prentice-Hall.

Brailovskaia, J., Rohmann, E., Bierhoff, H. W., \& Margraf, J. (2018). The brave blue world: Facebook flow and Facebook addiction disorder (FAD). PLoS One, 13(7), e0201484.

Brailovskaia, J., \& Margraf, J. (2017). Facebook addiction disorder (FAD) among German students - A longitudinal approach. PLoS One, 12(12), e0189719.

Brandenberg, G., Ozimek, P., Bierhoff, H.-W., \& Janker, C. (2019). The relation between use intensity of private and professional SNS, social comparison, self-esteem, and depressive tendencies in the light of self-regulation. Behaviour \& Information Technology, 1-14.

Brunstein, J. C., Dangelmayer, G., \& Schultheiss, O. C. (1996). Personal goals and social support in close relationships: Effects on relationship mood and marital satisfaction. Journal of Personality and Social Psychology, 71(5), 1006-1019.

Brunstein, J. C., \& Maier, G. W. (1996). Persönliche Ziele: Ein Überblick zum Stand der Forschung [Personal goals: A state-of-the-art review]. Psychologische Rundschau, 47, 146-160.

Brunstein, J. C., Schultheiss, O. C., \& Maier, G. W. (1999). The pursuit of personal goals. A motivational approach to well-being and life adjustment. In J. Brandtstädter \& R. M. Lerner (Eds.), Action and selfdevelopment. Theory and research through the life span (pp. 169196). Thousand Oaks: Sage.

Carver, C. S., \& Scheier, M. F. (1982). Control theory: A useful conceptual framework for personality-social, clinical, and health psychology. Psychological Bulletin, 92, 111-135.

Caruso, E. M., Baxter, B., Vohs, K. D., \& Waytz, A. (2013). Mere exposure to money increases endorsement of free-market capitalism and social inequality. Journal of Experimental Psychology: General, 142, 301-306.

Chou, H.-T. G., \& Edge, N. (2012). "They are happier and having better lives than I am": The impact of using Facebook on perceptions of others' lives. Cyberpsychology, Behavior and Social Networking, 15(2), 117-121.

Chu, S. C., Windels, K., \& Kamal, S. (2016). The influence of selfconstrual and materialism on social media intensity: A study of
China and the United States. International Journal of Advertising, 35(3), 569-588.

Cohen, J. (1988). Statistical power analysis for the behavioral sciences. 1988, Hillsdale, NJ: L. Lawrence Earlbaum Associates, 2.

Diener, E. D., Emmons, R. A., Larsen, R. J., \& Griffin, S. (1985). The satisfaction with life scale. Journal of Personality Assessment, 49(1), 71-75.

Ellison, N. B., Steinfield, C., \& Lampe, C. (2007). The benefits of Facebook "friends": Social capital and college students' use of online social network sites. Journal of Computer-Mediated Communication, 12, 1143-1168.

Engel, G. L. (1977). The need for a new medical model: A challenge for biomedicine. Science, 196(4286), 129-136.

Fenichel, M. (2009). Facebook addiction disorder (FAD). Retrieved on 22 December 2018 from http://www.fenichel.com/facebook/.

Festinger, L. (1954). A theory of social comparison processes. Human Relations, 7, 117-140.

Förster, J., \& Jostmann, N. B. (2015). What is automatic self-regulation? Zeitschrift für Psychologie, 220(3), 147-156.

Förster, J., \& Liberman, N. (2007). Knowledge activation. Social Psychology: Handbook of Basic Principles, 2, 201-231.

Förster, J., Liberman, N., \& Friedman, R. S. (2007). Seven principles of goal activation: A systematic approach to distinguishing goal priming from priming of nongoal constructs. Personality and Social Psychology Review, 11(3), 211e233.

Führer, A. \& Rohmann, E. (in preparation). Validierung einer deutschen Fassung der Difficulties in Emotion Regulation Scale.

Gibbons, F. X., \& Buunk, B. P. (1999). Individual differences in social comparison: Development of a scale of social comparison orientation. Journal of Personality and Social Psychology, 76(1), 129-142.

Gibbons, F. X., \& Gerrard, M. (1989). Effects of upward and downward social comparison on mood states. Journal of Social and Clinical Psychology, 8(1), 14.

Gonzales, A., \& Hancock, J. (2011). Mirror, mirror on my Facebook wall: Effects of exposure to Facebook on self-esteem. Cyberpsychology, Behavior and Social Networking, 14(1-2), 7983.

Gratz, K. L., \& Roemer, L. (2004). Multidimensional assessment of emotion regulation and dysregulation: Development, factor structure, and initial validation of the difficulties in emotion regulation scale. Journal of Psychopathology and Behavioral Assessment, 26(1), 4154.

Gross, J. J. (1998). The emerging field of emotion regulation: An integrative review. Review of General Psychology, 2(3), 271-299.

Gross, J. J., Richards, J. M., \& John, O. P. (2006). Emotion regulation in everyday life. In D. K. Snyder, J. A. Simpson, \& J. N. Hughes (Eds.), Emotion regulation in couples and families: Pathways to dysfunction and health (pp. 13-35). Washington, DC: American Psychological Association.

Gross, J. J., \& Thompson, R. A. (2007). Emotion regulation: Conceptual foundations. In J. J. Gross (Ed.), Handbook of emotion regulation (pp. 3-23). New York: Guilford Press.

Guimond, S., Branscombe, N. R., Brunot, S., Buunk, A. P., Chatard, A., Désert, M., Garcia, D. M., Haque, S., Martinot, D., \& Yzerbyt, V. (2007). Culture, gender, and the self: Variations and impact of social comparison processes. Journal of Personality and Social Psychology, 92(6), 1118-1134.

Hayes, A. F. (2013). Introduction to mediation, moderation, and conditional process analysis. A Regression-Based Approach. New York: Guilford Press.

Higgins, E. T., Klein, R., \& Strauman, T. (1985). Self-concept discrepancy theory: A psychological model for distinguishing among different aspects of depression and anxiety. Social Cognition, 3, 51-76.

Higgins, E. T., Strauman, T., \& Klein, R. (1986). Standards and the process of self-evaluation: Multiple affects from multiple stages. In R. M. Sorrentino \& E. T. Higgins (Eds.), Handbook of motivation 
and cognition: Foundations of social behavior (pp. 23-63). New York: Guilford Press.

Hormes, J. H., Kearns, B., \& Timko, C. A. (2014). Craving Facebook? Behavioral addiction to online social networking and its association with emotion regulation deficits. Addiction, 109(12), 2079-2088.

Kamal, S., Chu, S. C., \& Pedram, M. (2013). Materialism, attitudes, and social media usage and their impact on purchase intention of luxury fashion goods among American and Arab young generations. Journal of Interactive Advertising, 13(1), 27-40.

Kim, J., \& Lee, J. E. R. (2011). The Facebook paths to happiness: Effects of the number of Facebook friends and self-presentation on subjective well-being. CyberPsychology, behavior, and social networking, 14(6), 359-364.

Kross, E., Verduyn, P., Demiralp, E., Park, J., Lee, D. S., Lin, N., Shablack, H., Jonides, J., \& Ybarra, O. (2013). Social Facebook use predicts declines in subjective well-being in young adults. PLoS One, 8(8), e69841.

Kruglanski, A. W., Shah, S. Y., Fishbach, A., Friedman, R., Chun, W. Y., \& Sleeth-Keppler, D. (2002). A theory of goal systems. Advances in Experimental Social Psychology, 34, 331-378.

Lee, S. Y. (2014). How do people compare themselves with others on social network sites? The case of Facebook. Computers in Human Behavior, 31, 253-260.

Lewin, K. (1935). A dynamic theory of personality: Selected papers (DK Adams \& KE Zener, Trans. New York: McGraw.

Locke, E. A. (2001). Setting goals for life and happiness. In C. R. Snyder \& S. J. Lopez (Eds.), Handbook of positive psychology (pp. 299 312). New York: Oxford.

Lockwood, P., \& Kunda, Z. (1997). Superstars and me: Predicting the impact of role models on the self. Journal of Personality and Social Psychology, 73, 91-103.

Masters, J. C. (1991). Strategies and mechanisms for the personal and social control of emotion. In J. Garber \& K. A. Dodge (Eds.), The development of emotion regulation and dysregulation (pp. 182207). Cambridge, UK: Cambridge University Press.

McAndrew, F. T., \& Jeong, H. S. (2012). Who does what on Facebook? Age, sex, and relationship status as predictors of social Facebook use. Computers in Human Behavior, 28, 2359-2365.

Mehdizadeh, S. (2010). Self-presentation 2.0: Narcissism and self-esteem on Facebook. Cyberpsychology, Behavior and Social Networking, 13(4), 357-364.

Mischel, W., \& Ayduk, O. (2004). Willpower in a cognitive-affectiveprocessing system: The dynamics of delay of gratification. In R. F. Baumeister \& K. D. Vohs (Eds.), Handbook of self-regulation: Research, theory, and applications (pp. 99-129). New York: Guilford Press.

Nabi, R. L., Prestin, A., \& So, J. (2013). Facebook friends with (health) benefits? Exploring social network site use and perceptions of social support, stress, and well-being. Cyberpsychology, Behavior and Social Networking, 16(10), 721-727.

Nadkarni, A., \& Hofmann, S. G. (2012). Why do people use Facebook? Personality and Individual Differences, 52, 243-249.

Ozimek, P., Baer, F., \& Förster, J. (2017). Materialists on Facebook: The self-regulatory role of social comparisons, objectification and instrumentalization of Facebook friends. Helivon, 3, e00449.

Ozimek, P., \& Bierhoff, H.-W. (2016). Social Facebook use depending on age: The influence of social comparisons. Computers in Human Behavior, 61, 271-279.

Ozimek, P., Bierhoff, H.-W., \& Hanke, S. (2018). Do vulnerable narcissists profit more from social Facebook use than grandiose narcissists? An examination of narcissistic social Facebook use in the light of self-regulation and social comparison theory. Personality and Individual Differences, 124, 168-177.

Ozimek, P., \& Förster, J. (2017). The impact of self-regulatory states and traits on social Facebook use: Priming materialism and social comparisons. Computers in Human Behavior, 71, 418-427.
Papies, E. K., \& Hamstra, P. (2010). Goal priming and eating behavior: Enhancing self-regulation by environmental cues. Health Psychology, 29, 384-388.

Rohmann, E., \& Bierhoff, H. W. (2016). Optimismus und positive Illusionen [optimism and positive illusions]. In H. W. Bierhoff \& D. Frey (Eds.), Selbst und soziale Kognition Encyklopedia of psychology, series social psychology (Vol. 1, pp. 471-508). Göttingen: Hogrefe.

Roth, P. (2018). Nutzerzahlen: Facebook, Instagram, Messenger und WhatsApp, Highlights, Umsätze, uvm. (Stand April 2018). Retrieved on 13 May 2018 from https://allfacebook.de/toll/stateof-facebook.

Ryan, T., Chester, A., Reece, J., \& Xenos, S. (2014). The uses and abuses of Facebook: A review of Facebook addiction. Journal of Behavioral Addictions, 3(3), 133-148.

Ryan, T., \& Xenos, S. (2011). Who uses Facebook? An investigation into the relationship between the big five, shyness, narcissism, loneliness, and Facebook usage. Computers in Human Behavior, 27, $1658-1664$.

Santas-Atalar, D., Gencöz, T., \& Özen, A. (2015). Confirmatory factor analyses of the difficulties in emotion regulation scale (DERS) in a Turkish adolescent sample. European Journal of Psychological Assessment, 31, 12-19.

Schneider, S., \& Schupp, J. (2011). The social comparison scale: Testing the validity, reliability, and applicability of the IOWA-Netherlands comparison orientation measure (INCOM) on the German population. Data Documentation, 55.

Schumacher, J. (2003). SWLS-satisfaction with life scale. Diagnostische Verfahren zu Lebensqualität und Wohlbefinden, 305, 309.

Steers, M.-L. N., Wickham, R. E., \& Acitelli, L. K. (2014). Seeing everyone else's highlight reels: How Facebook usage is linked to depressive symptoms. Journal of Social and Clinical Psychology, 33(8), 701-731.

Steinfield, C., Ellison, N. B., \& Lampe, C. (2008). Social capital, selfesteem, and use of online social network sites: A longitudinal analysis. Journal of Applied Developmental Psychology, 29, 434-445.

Toma, C. L. (2013). Feeling better but doing worse: Effects of Facebook self-presentation on implicit self-esteem and cognitive task performance. Media Psychology, 16(2), 199-220.

Valkenburg, P. M., Schouten, A. P., \& Peter, J. (2005). Adolescents' identity experiments on the internet. New Media and Society, 7(3), 282-402.

Valkenburg, P. M., Schouten, A. P., \& Peter, J. (2006). Friend networking sites and their relationship to adolescents' well-being and selfesteem. Cyperpsychology, Behavior, and Social Networking, 9(5), 584-590.

Verduyn, P., Ybarra, O., Résibois, M., Jonides, J., \& Kross, E. (2017). Do social network sites enhance or undermine subjective well-being? A critical review. Social Issues and Policy Review, 11(1), 274-302.

Vogel, E. A., Rose, J. P., Okdie, B. M., Eckles, K., \& Franz, B. (2015). Who compares and despairs? The effect of social comparison orientation on social media use and its outcomes. Personality and Individual Differences, 86, 249-256.

Wills, T. A. (1981). Downward comparison principles in social psychology. Psychological Bulletin, 90(2), 245-271.

Wilson, R. E., Gosling, S. D., \& Graham, L. T. (2012). A review of Facebook research in the social sciences. Perspectives on Psychological Science, 7(3), 203-220.

Zerback, T., Schoen, H., Jackob, N., \& Schlereth, S. (2009). Zehn Jahre Sozialforschung mit dem Internet - eine Analyse zur Nutzung von Online-Befragungen in den Sozialwissenschaften. In Sozialforschung im Internet (pp. 15-31). Wiesbaden: VS Verlag für Sozialwissenschaften.

Zuckerberg, M. (2017). Bringing the world closer together. Retrieved on 03 January 2019 from https://www.facebook.com/notes/mark- 